\title{
Ultrafast Surface-Enhanced Raman Probing of the Role of Hot Electrons in Plasmon-Driven Chemistry
}

\section{Supporting Information}

\author{
Nathaniel C. Brandt, Emily L. Keller, and Renee R. Frontiera
}

\section{Methods}

\section{Sample preparation}

$\mathrm{AgNO}_{3}$, sodium citrate, and 4-NBT for sample preparation were obtained from Sigma-Aldrich and used without further purification. To synthesize colloidal AgNPs, $87.8 \mathrm{mg}$ of $\mathrm{AgNO}_{3}$ dissolved in $490 \mathrm{~mL}$ of nanopure $\mathrm{H}_{2} \mathrm{O}$ was combined with $11.5 \mathrm{~mL}$ $1 \%$ by weight aqueous sodium citrate dihydrate under heat and stirring. ${ }^{1}$ A $230 \mu \mathrm{L}$ aliquot of the as-synthesized solution of AgNPs was placed in a $1.7 \mathrm{~mL}$ microcentrifuge tube to which $70 \mu \mathrm{L}$ of a saturated 4-NBT aqueous solution was added and mixed by vortexing. The concentration of the saturated 4-NBT solution was measured by UVvisible spectroscopy to be approximately $200 \mu \mathrm{M}$, well in excess of that required for the formation of a monolayer of 4-NBT on the AgNP substrates. After approximately 10 minutes, $100 \mu \mathrm{L}$ of a $0.1 \mathrm{M}$ aqueous solution of $\mathrm{NaCl}$ was added to induce aggregation, after which the sample was again mixed by vortexing. The sample was transferred to a 2 mm pathlength quartz cuvette (Starna 21-Q-2) for all measurements. Samples were stirred throughout the course of the experiment to refresh the sample in the irradiated volume and to minimize precipitation of the aggregated particles.

\section{Ultrafast SERS instrumentation}

Ultrafast SERS measurements were performed using the instrument depicted schematically in Fig. S1. The instrument utilized a Yb-doped fiber-based amplified laser (Clark-MXR Impulse), which produced $<250$ fs pulses centered at $1035 \mathrm{~nm}$ at $24.5 \mathrm{MHz}$ 
repetition rate, with an average power of $20.5 \mathrm{~W}$. To generate the picosecond probe pulse, $6 \mathrm{~W}$ of the incident beam entered a spectral filter constructed in a retroreflecting geometry ${ }^{2}$, which contained a transmission grating (LightSmyth Technologies LSFSG1000-3212-94) to disperse the beam and a $100 \mathrm{~mm}$ focal length cylindrical lens (Newport CKX100AR.16) to image the beam onto a slit. The spectral width of the filtered output was $13.4 \mathrm{~cm}^{-1}$, as measured from the $801 \mathrm{~cm}^{-1}$ peak of cyclohexane. For the pump beam, $3 \mathrm{~W}$ of the fundamental laser output was focused with a $200 \mathrm{~mm}$ focal length lens (Thorlabs LA1253-B) onto a $3 \mathrm{~mm}$ beta-barium borate crystal (Newlight Photonics) to obtain a $518 \mathrm{~nm}$ pulse, which was collimated with a $200 \mathrm{~mm}$ focal length lens (Thorlabs LA1253-YAG). The pump beam was reflected from two longpass dichroic mirrors (Thorlabs DMLP900 and DMLP950) and passed through a shortpass transmission filter (Thorlabs FES0950) to remove any remaining $1035 \mathrm{~nm}$ light, and the polarization was rotated to match the horizontal polarization of the probe beam using a half wave plate (Thorlabs AHWP05M-980). A 100 mm focal length achromatic lens (Edmund Optics 49374) focused the pump and probe beams onto the sample to a spot size of approximately 80 microns. The probe power was $16.8 \mathrm{~mW}$ and the pump power ranged from 5 to 50 $\mathrm{mW}$. The time resolution of the instrument was $2.7 \mathrm{ps}$, which was determined from an optical Kerr effect measurement of neat cyclohexane at the sample position. After the sample, an $850 \mathrm{~nm}$ shortpass filter (Thorlabs FGL850) blocked pump transmission, and a $60 \mathrm{~mm}$ focal length lens (Thorlabs, LA1134-C) collimated the spontaneous Raman signal. An additional longpass filter (Thorlabs FGL830) was used to further reduce pump beam scatter. A $100 \mathrm{~mm}$ focal length achromatic lens (Thorlabs, AC254-100-C) focused the Raman signal into a $0.3 \mathrm{~m}$ spectrograph (Princeton Instruments SP2300i) equipped 
with a $600 \mathrm{gr} / \mathrm{mm}, 750 \mathrm{~nm}$ blaze grating. A $1064 \mathrm{~nm}$ Raman filter (Edmund Optics 47510) directly before the spectrograph removed any remaining $1035 \mathrm{~nm}$ light from the Raman signal. A 1024 pixel, liquid nitrogen cooled InGaAs photodiode array (Princeton Instruments PyLoN-IR 1.7) was used for detection of the Stokes-shifted spontaneous Raman scattering.

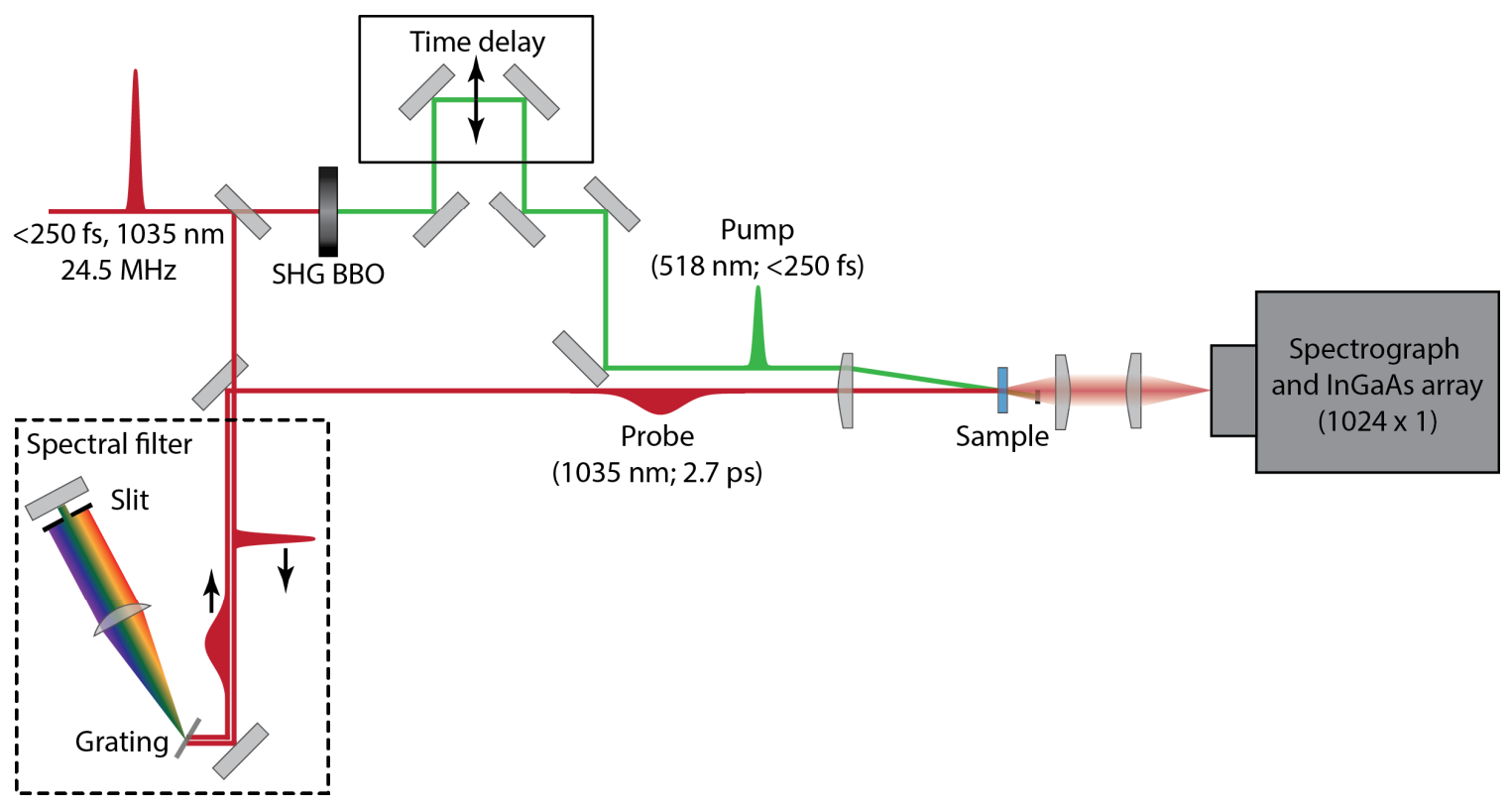

Figure S1. Schematic depiction of the ultrafast SERS instrument. Acronyms used in the figure are second harmonic generation (SHG) and beta-barium borate (BBO).

\section{Data collection and processing}

Each ultrafast SER spectrum was obtained from 5 averaged frames that were integrated for 10 seconds each. For pump-probe measurements, a delay stage (Newport XMS50) in the pump path allowed for data acquisition at each pump/probe delay point $\tau$, which were accessed in a randomized order. A spectrum with the pump pulse blocked by a mechanical shutter (denoted as $S_{o f f}(\tau)$ ) was taken followed immediately by a spectrum with the pump pulse present (denoted as $S_{o n}(\tau)$ ) at each time point. After the spectra were averaged, the $S_{\text {off }}(\tau)$ spectrum was subtracted from the $S_{\text {on }}(\tau)$ spectrum to isolate the 
effects of the pump pulse at different time delays. However, the large amount of static background from the propagation of the pump beam through the highly scattering AgNP solution made the difference spectra from this subtraction difficult to interpret. As a result, a second subtraction step was done, in which the difference spectrum at $\tau=-50 \mathrm{ps}$ was subtracted from all other difference spectra, to give a final "double difference" spectrum $S_{d d}(\tau)$, given by

$$
S_{d d}(\tau)=S_{o n}(\tau)-S_{o f f}(\tau)-\left[S_{o n}(-50 \mathrm{ps})-S_{o f f}(-50 \mathrm{ps})\right]
$$

for each pump/probe delay time $\tau$. At $\tau=-50 \mathrm{ps}$, the probe pulse arrives well before the pump pulse, thus the difference spectrum at this time delay only shows the static background from the pump without any sample dynamics due to excitation.

\section{Data fitting}

Processed SERS double-difference spectra for each pump/probe time delay were fit to sums of multiple Fano lineshapes using a custom-written Igor Pro fitting routine. The fits for the region that includes the 1077 and $1139 \mathrm{~cm}^{-1}$ features, from which the lifetime fit in Fig. 3b in the main text was generated, appear below in Fig. S2. Masked areas of data, which were ignored in the fitting routine, are also noted in grey.

To extract lifetime information, the fitted Fano amplitude of the $1139 \mathrm{~cm}^{-1}$ feature was fit to a convolution of a Gaussian and an exponential decay, with the width of the Gaussian held to the measured cross-correlation response of the instrument. Fit parameters for this mode at all times delays are shown in Table S1. 


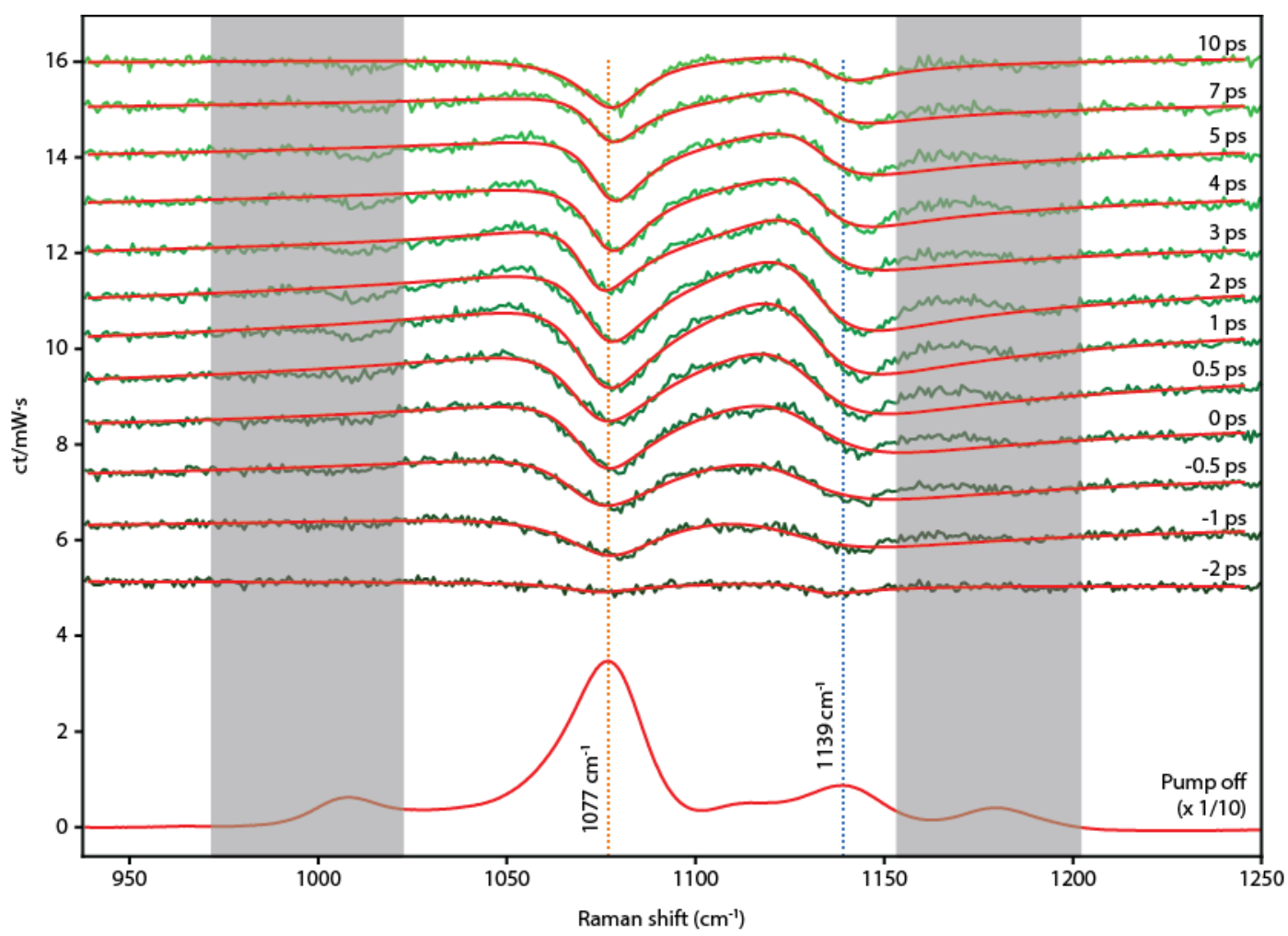

Figure S2. Fitted ultrafast SER double-difference spectra relative to SER spectrum collected with pump pulse blocked. Gray rectangles denote masked areas, which were ignored during fitting. 

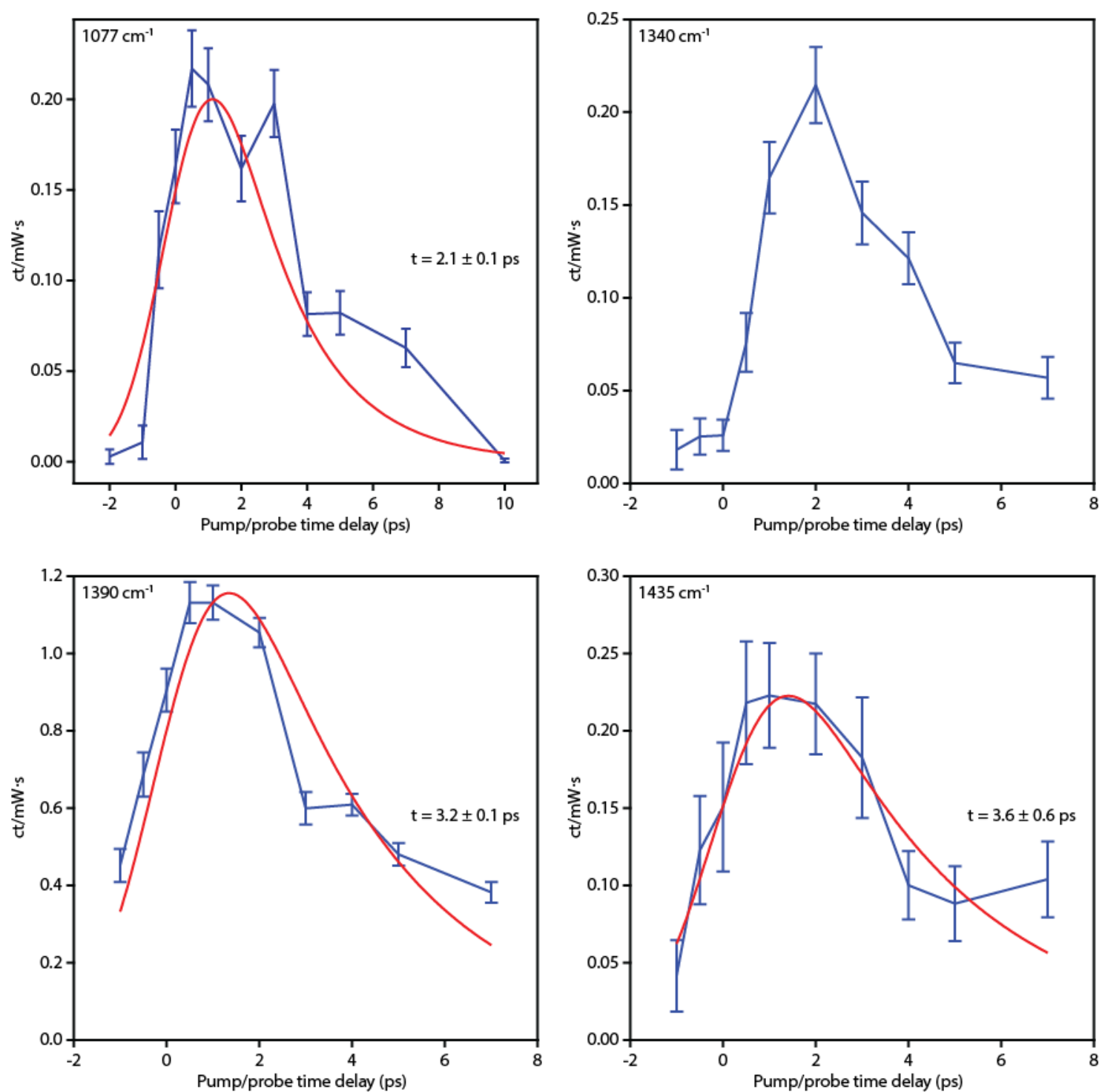

Figure S3. Convolved fits (-) to Fano-fitted amplitudes (-) of several ultrafast SERS features, giving lifetimes for the transient Fano features that are in good agreement with the $3.9 \pm 0.1 \mathrm{ps}$ lifetime obtained for the $1139 \mathrm{~cm}^{-1}$ feature described in the main text. Note that the $1340 \mathrm{~cm}^{-1}$ feature was not satisfactorily fit to the convolution function used in the other fits.

\begin{tabular}{|c|c|c|c|}
\hline Time point $(\mathrm{ps})$ & Amplitude $(\mathrm{ct} / \mathrm{mW} \mathrm{s})$ & Frequency $\left(\mathrm{cm}^{-1}\right)$ & $\mathrm{q}$ \\
\hline-2 & $-0.03 \pm 0.02$ & $1133 \pm 2$ & $2.3 \pm 0.6$ \\
\hline-1 & $-0.33 \pm 0.05$ & $1126 \pm 2$ & $1.1 \pm 0.1$ \\
\hline-0.5 & $-0.57 \pm 0.04$ & $1126 \pm 1$ & $0.81 \pm 0.06$ \\
\hline 0 & $-0.75 \pm 0.04$ & $1130 \pm 1$ & $0.77 \pm 0.05$ \\
\hline 0.5 & $-0.93 \pm 0.04$ & $1129.1 \pm 0.7$ & $0.78 \pm 0.03$ \\
\hline 1 & $-1.02 \pm 0.04$ & $1130.2 \pm 0.6$ & $0.82 \pm 0.03$ \\
\hline
\end{tabular}




\begin{tabular}{|c|c|c|c|}
\hline 2 & $-0.93 \pm 0.03$ & $1131.3 \pm 0.6$ & $0.85 \pm 0.03$ \\
\hline 3 & $-0.78 \pm 0.03$ & $1130.7 \pm 0.7$ & $0.69 \pm 0.03$ \\
\hline 4 & $-0.60 \pm 0.03$ & $1132.5 \pm 0.7$ & $0.92 \pm 0.04$ \\
\hline 5 & $-0.53 \pm 0.03$ & $1132.7 \pm 0.8$ & $0.89 \pm 0.05$ \\
\hline 7 & $-0.40 \pm 0.02$ & $1133.0 \pm 0.8$ & $0.91 \pm 0.05$ \\
\hline 10 & $-0.11 \pm 0.02$ & $1136.4 \pm 0.9$ & $1.9 \pm 0.2$ \\
\hline
\end{tabular}

Table S1. Fano fit parameters for the $1139 \mathrm{~cm}^{-1}$ mode as function of time delay.

\section{Discussion}

\section{Power dependence}

To determine the scaling of the transient Fano effect with variations in excitation power, the average power of the pump beam was varied using a variable neutral density filter. The results appear in Figures S4-6, in which the data have been normalized to account for variations in the amplitude of the pump-off spectra at each value of the average pump power due to increased pump-induced damage at higher pump power. The transient Fano amplitude at $1139 \mathrm{~cm}^{-1}$ exhibited a linear dependence on the average power of the pump beam at low excitation power. The Fano q parameter, a measure of the dispersion of the line, was relatively unchanged at all time points and powers probed, except for values for low power experiments at time points well before or after time zero, when the small amplitude of the transient Raman features leads to large errors in the fitting.

At sufficiently high excitation power, the transient Fano amplitude deviated significantly from the linear dependence observed at lower excitation power. This can be seen in Fig. S4 when the average excitation power $P$ is increased to $2 P_{0}$, where $P_{0}$ represents the average excitation power used for the measurements presented in the main 
text. The underlying causes of the deviation from linearity warrant further investigation and were beyond the scope of our present investigation; however, the most likely cause is degradation of the AgNP substrate at high laser irradiance. Other possible causes include saturation of either plasmon excitations or hot electron production in the AgNP substrates, or the availability of an alternative hot electron loss pathway at higher excitation power.

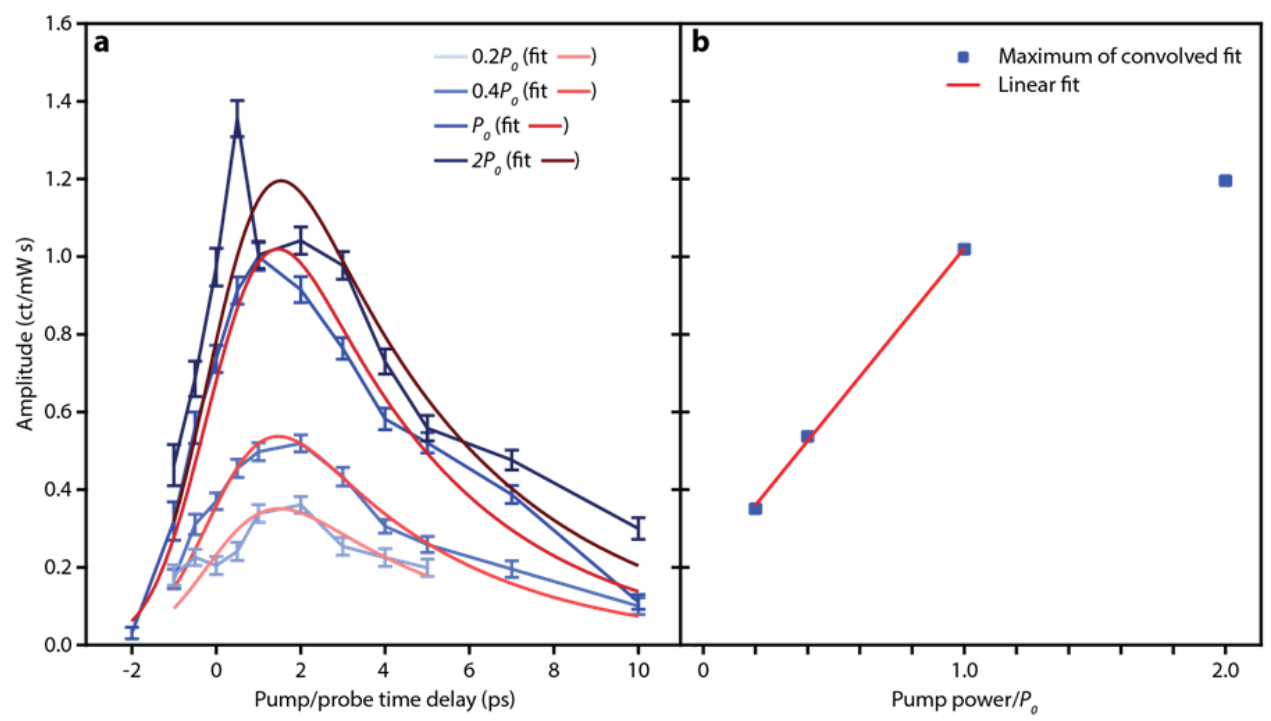

Figure S4. (a) Dependence of the $1139 \mathrm{~cm}^{-1}$ transient Fano amplitude and convolved amplitude fit on variations in the average power in the pump beam, denoted as multiples of the incident power $P_{0}=25 \mathrm{~mW}$ used for the measurements presented in the main text. (b) Maximum of the convolved fit of the $1139 \mathrm{~cm}^{-1}$ Fano amplitude as a function of pump average power, with a linear fit to the data from $P=0.2 P_{0}$ to $P=P_{0}$. 


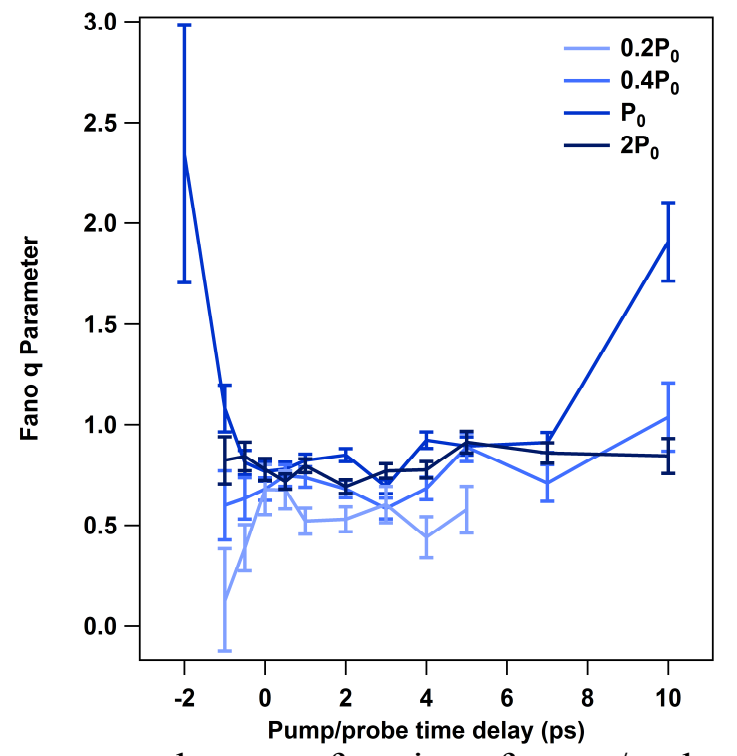

Figure S5. Fano q parameter values as a function of pump/probe time delay for a range of powers, where $\mathrm{P}_{0}=25 \mathrm{~mW}$.

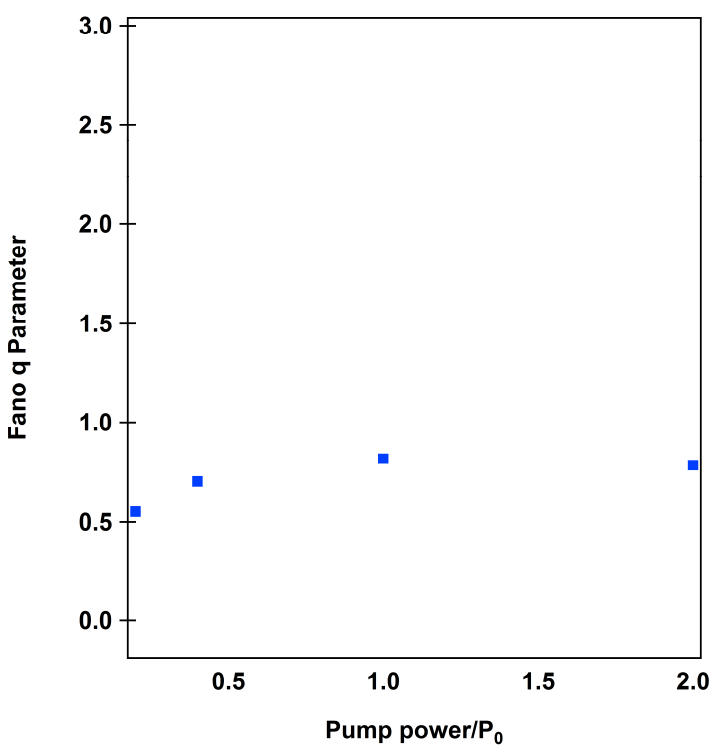

Figure S6. Fano q parameter as a function of power. This plot was made by averaging the $\mathrm{q}$ value for timepoints between -1 and $5 \mathrm{ps}$.

\section{References}

(1) Lee, P. C.; Meisel, D. Adsorption and Surface-Enhanced Raman of Dyes on Silver and Gold Sols. J.Phys.Chem. 1982, 86 (17), 3391-3395.

(2) Shim, S.; Mathies, R. A. Generation of Narrow-Bandwidth Picosecond Visible Pulses from Broadband Femtosecond Pulses for Femtosecond Stimulated Raman. Appl. Phys. Lett. 2006, 89 (12), 121124. 